\title{
BER ANALYSIS FOR DOWNLINK MIMO-NOMA SYSTEMS OVER RAYLEIGH FADING CHANNELS
}

\author{
Vu Tran Hoang Son ${ }^{1,2}$ and Dang Le Khoa ${ }^{1,2 *}$ \\ ${ }^{1}$ VNUHCM - University of Science, Ho Chi Minh City, Vietnam \\ ${ }^{2}$ Vietnam National University, Ho Chi Minh City, Vietnam
}

\begin{abstract}
The Multiple-input multiple-output (MIMO) technique combined with non-orthogonal multiple access (NOMA) has been considered to enhance total system performance. This paper studies the bit error rate of two-user power-domain NOMA systems using successive interference cancellation receivers, with zeroforcing equalization over quasi-static Rayleigh fading channels. Successive interference cancellation technique at NOMA receivers has been the popular research topic due to its simple implementation, despite its vulnerability to error propagation. Closed-form expressions are derived for downlink NOMA in single-input single-output and uncorrelated quasi-static MIMO Rayleigh fading channel. Analytical results are consolidated with Monte Carlo simulation.
\end{abstract}

\section{KEYWORDS}

Bit error rate, Multiple-input multiple-output, non-orthogonal multiple access.

\section{INTRODUCTION}

Recent communication systems aim to meet the high capacity requirements. Non-orthogonal multiple access (NOMA) is not only available in conventional $4 \mathrm{G}$, recommended in $5 \mathrm{G}$ and future $6 \mathrm{G}$ due to many outstanding benefits $[1,2]$. NOMA can be divided into two main categories as power-domain NOMA and code-domain NOMA. Successive interference cancellation (SIC) is well-known in power-domain NOMA systems for its simple implementation, although it has low performance compared to other detection techniques such as using log-likelihood ratios[3], or machine learning[4, 5]. There have been many studies in the probability of outage[6], power allocation[7], system capacity [8], bit error rate (BER)[9-13], showing that NOMA is superior to conventional orthogonal multiple access[14]. NOMA can be integrated with multiple input, multiple output(MIMO), which can result in a notable increase in capacity[15], or even combined with deep neural network and beam forming [16]. To the best of the authors' knowledge, there is no approach to BER expressions when changing between multiuser superposition transmission (MUST) categories in 3GPP LTE Release $13[17,18]$ to generate NOMA signals. The main contributions of this paper are:

1) Closed-form BER expressions for NOMA in two-user scenario with quadrature phase shift keying(QPSK) modulation over quasi-static single-input single-output (SISO) Rayleigh fading channels are investigated.

2) Under certain conditions, BER expressions perfectly suit the MIMO case. This is verified via Monte Carlo simulation.

3) When Rayleigh channel has multiple taps, simulations barely fit analytical results due to the limited response of zero-forcing $(\mathrm{ZF})$ equalization. Because of this, via simulations, authors have combined the conventional systems with orthogonal frequency division 
International Journal of Computer Networks \& Communications (IJCNC) Vol.13, No.6, November 2021

multiplexing (OFDM) to reduce inter-symbol interference (ISI), regardless of nonlinearities.

From this section, the paper is organized as follows. Section 2 presents and compares with related work in the same field. Section 3 introduces the system model and the corresponding parameters. Based on preexisting papers, Section 4 analyzes BER in MUST category 1. Section 5 proceeded to MUST category 2 with a different approach, giving unified results when applying in MUST category 1. Section 6 considered the effect of MIMO in NOMA systems, under certain constraints, compared to SISO. All expressions are verified in Section 7 via simulations, and conclusions along with future works are drawn in Section 8.

\section{RELATED WORK}

BER is one of the key factors to evaluate MIMO-NOMA system performance. This section will consider related work that analyzes BER in the NOMA system. In [9], BER expressions of uplink NOMA with SIC receivers has been derived over Additive White Gaussian Noise(AWGN) channels. In [10], F. Kara has developed BER expressions for downlink NOMA over Rayleigh fading channels with two users: far user (FU) using binary phase shift keying (BPSK), and near user (NU) using QPSK. All expressions are followed by simulations. With significant effort, T. Assaf et al. have introduced analytical BER through Nakagami-m fading channels in scenarios of two and three users with QPSK modulation in [11], and through AWGN channels with general M-QAM modulation[13].The authors in [12] investigated BER in QPSK-modulated NOMA users on the AWGN channel and verified by software defined radio platforms. Similar research in recent papers shows that NOMA signals are generated using only MUST type 1 and are limited in SISO systems. Moreover, in the following years, applications of MIMO, or even massive MIMO (m-MIMO), will be in new generation mobile networks. Our research is to find out the BER analysis approach united in both MUST type 1 and type 2 in SISO systems, propose a comparison with MIMO in Rayleigh fading channels, using Monte Carlo simulations to validate the results.

\section{SYSTEM MODEL}

\subsection{Downlink NOMAsystem}

Consider downlink SISO-NOMA system with two user's equipment (UEs) and a base station (BS) as depicted in Figure 1.

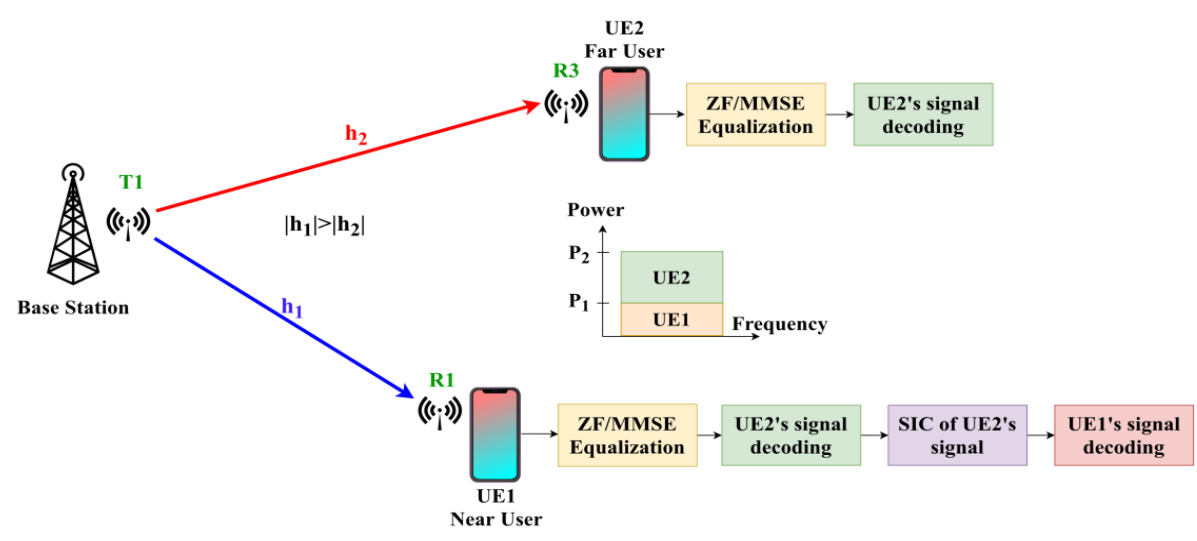

Figure 1. Downlink SISO-NOMA system 
International Journal of Computer Networks \& Communications (IJCNC) Vol.13, No.6, November 2021

BS will generate NOMA signals using MUST cat.1 or MUST cat.2 [17, 18]. Based on Figure 1, the generated signal $x_{s c}$ can be expressed as

$$
x_{s c}=\sqrt{P_{1}} x_{1}+\sqrt{P_{2}} x_{2},
$$

where $x_{k}$ and $P_{k}$ are respectively $k$ th user's signals and power allocation coefficients using QPSK modulation, with BS transmitted power $P_{S}$ and power allocation ratio $\alpha$ satisfies $P_{S}=P_{1}+P_{2}, P_{1}=\alpha P_{S}, P_{2}=(1-\alpha) P_{S}$. For users' fairness, near user (NU) UE1 will be allocated less power than far user (FU) UE2, which means $P_{1}<P_{2}$ or $0<\alpha<0.5$.

For quasi-static fading channel, the received signal at user $k$ th can be described as[19, 20]

$$
\mathbf{y}_{k}=\mathbf{h}_{k} * \mathbf{x}_{s c}+\mathbf{n}_{k},
$$

where $\mathbf{y}_{k}$ is the received signal matrix, $\mathbf{h}_{k}$ is the uncorrelated quasi-static Rayleigh channel matrix with $\mathbf{h}_{k} \square \mathrm{CN}\left(0, g_{k}\right), g_{k}$ is the average channel gain $g_{k}=\mathrm{E}\left[\left|\mathbf{h}_{k}\right|^{2}\right], \mathbf{n}_{k}$ is circularly symmetric white Gaussian noise with $\mathbf{n}_{k} \square \mathrm{CN}\left(0, N_{0}\right)$, "** is the convolution operator which can be reduced to inner product when the channel is single-tap, E[.] is the expected value, ||$^{2}$ is the Euclidean norm.

SIC process will be conducted at NU. NU will estimate FU's symbols and use SIC to eliminate interference by subtracting FU's symbols from the received signal, then NU decodes its own symbols. On the other hand, FU does not need to apply SIC, instead detect its symbols by considering others as noise.

\subsection{MIMO-NOMA system}

One of the techniques in MIMO is spatial division multiplexing (SDM), which takes advantage of the multipath channel to enhance system capacity without increasing the bandwidth. Figure 2 presents a downlink MIMO-NOMA system using SDM 2x2.

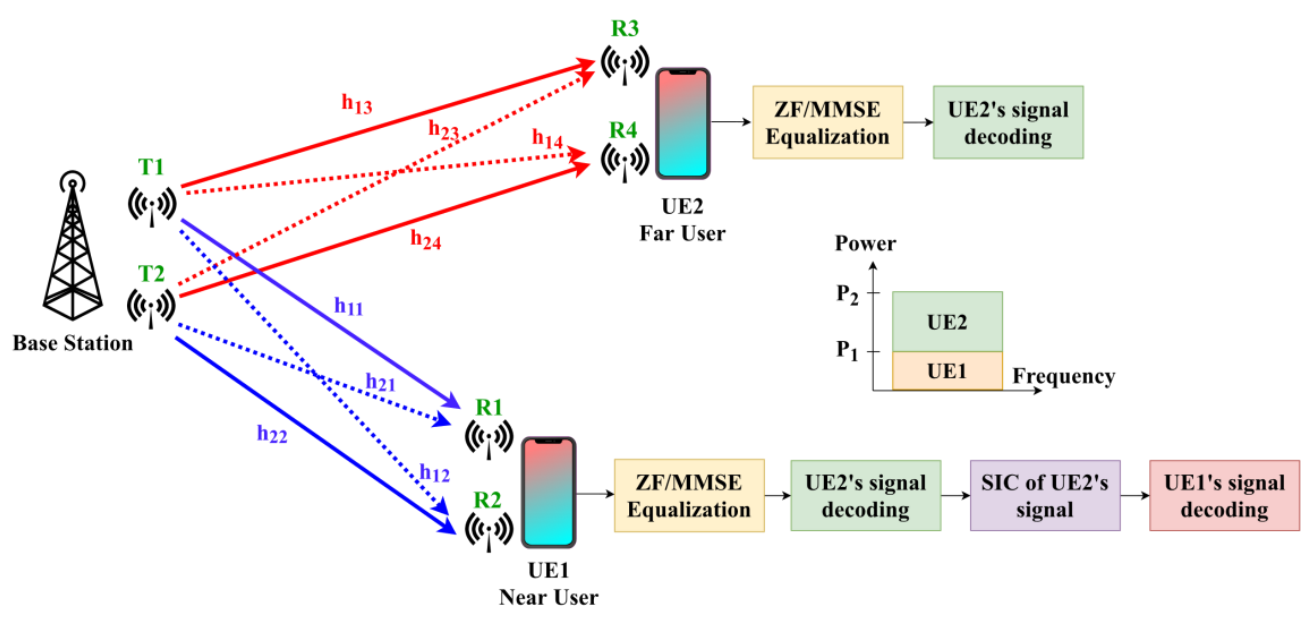

Figure 2. Downlink MIMO-NOMA system 


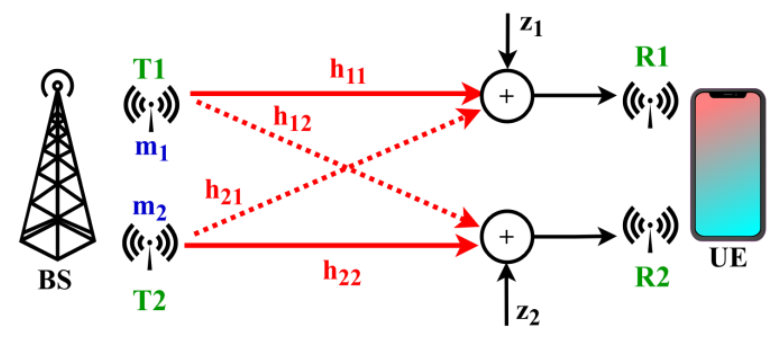

Figure 3. MIMO SDM 2x2 system

If the spacing between the transmitting and receiving antennas is large enough, the scattering characteristics will be uncorrelated, so each path is independent. Under these conditions, the desired signal from the target transmitting antenna can be detected by treating other transmitted signals as interferences. This is called linear signal detection. We will concentrate on the technique that nullifies the interference, zero-forcing method. From Figure 3, the received signal matrix can be expressed as [21]

$$
\overline{\mathbf{y}}=\overline{\mathbf{h}} \mathbf{m}+\mathbf{z}=\overline{\mathbf{h}}_{1} m_{1}+\overline{\mathbf{h}}_{2} m_{2}+\mathbf{z},
$$

where $\overline{\mathbf{h}}$ denotes an uncorrelated MIMO 2x2 Rayleigh channel matrix with $(j, i)$ th entry $h_{j i}$ for the single-tap channel gain between the $i$ th transmit antenna and the $j$ th receive antenna, $j=1,2$ and $i=1,2$, then $\overline{\mathbf{h}}=\left[\begin{array}{ll}h_{11} & h_{21} \\ h_{12} & h_{22}\end{array}\right]$.The spatially multiplexed data and the corresponding received signals in a symbol time are represented by $\mathbf{m}=\left[m_{1}, m_{2}\right]^{T}$ and $\overline{\mathbf{y}}=\left[\bar{y}_{1}, \bar{y}_{2}\right]^{T}$, respectively, where $m_{i}$ is the message from the $i$ th transmit antenna, $\bar{y}_{j}$ is the received signal at the $j$ th receive antenna, $[.]^{T}$ is the matrix transpose operation. The transmission power of each antenna is assumed to be one. Let $\mathbf{z}=\left[z_{1}, z_{2}\right]^{T}$ with $z_{j}$ denote the circularly symmetric white Gaussian noise with a variance of $\sigma_{z}^{2}$ at the $j$ th receive antenna, and $\overline{\mathbf{h}}_{i}$ denote the $i$ th column vector of the channel matrix $\overline{\mathbf{h}}$.

The effect of the channel is inverted by a weight matrix $\mathbf{w}$ that $\tilde{\mathbf{m}}=\mathbf{w} \overline{\mathbf{y}}=\left[\tilde{m}_{1}, \tilde{m}_{2}\right]^{T}$, therefore, we can detect the desired signals from each antenna. The $\mathrm{ZF}$ technique nullifies the interference by the weight matrix $\mathbf{w}_{Z F}=\left(\overline{\mathbf{h}}^{H} \overline{\mathbf{h}}\right)^{-1} \overline{\mathbf{h}}^{H}$ with [.] $]^{H}$ is the Hermitian transpose operation. All formulas here can be used in the time domain and inner product because of single-tap channel assumptions, but when the channel is multiple-tap, all quantities need to be in the frequency domain via the Fourier transform. Received signals after ZF equalization is

$$
\begin{aligned}
\tilde{\mathbf{m}} & =\mathbf{w}_{Z F} \overline{\mathbf{y}} \\
& =\mathbf{m}+\left(\overline{\mathbf{h}}^{H} \overline{\mathbf{h}}\right)^{-1} \overline{\mathbf{h}}^{H} \mathbf{z} \\
& =\mathbf{m}+\widetilde{\mathbf{z}}_{\mathbf{Z F}} .
\end{aligned}
$$


As seen in (3.4), each original data stream is separated from the multiplexed signal. After equalization, every UE (except the strongest one) detects its information using SIC process. Notice that if the channel has only one tap, a complex $\overline{\mathbf{h}}$ can be presented in the form of amplitude and phase, such for SISO case that $\overline{\mathbf{h}}=\bar{h}=\bar{h}_{R}+\bar{j} \bar{h}_{I}=|\bar{h}| \exp (\bar{j} \theta)$ with $\bar{j}^{2}=-1$, $|\bar{h}|=\sqrt{\bar{h}_{R}^{2}+\bar{h}_{I}^{2}}$ is the magnitude of $\bar{h}, \theta$ is the argument of $\bar{h}$, then ZF can be implemented by[10,21]

$$
\begin{aligned}
\frac{y}{\bar{h}} & =\frac{y}{|\bar{h}|^{2}} \operatorname{conj}(\bar{h}) \\
& =\frac{y}{|\bar{h}|^{2}}|\bar{h}| \exp (-\bar{j} \theta) \\
& =x+\frac{n}{|\bar{h}|} \exp (-\bar{j} \theta) .
\end{aligned}
$$

From (3.5), $n$ and $n \exp (-\bar{j} \theta)$ is different by a phase shift, however, $n$ is circularly symmetric so $n$ and $n \exp (-\bar{j} \theta)$ have the same probability distribution. This is one of the key factors to form the composite reference constellation discussed later in Section 4 and Section 0.

\section{BER IN MUST CATEGORY 1}

Based on (3.5), Figure 4shows the reference constellation to detect signals at each UE when using MUST category 1 . Red bits denote FU's bits, and black bits are NU's bits. The order of bits is ascending, where the most significant bit is leftmost and is stated as the first bit.

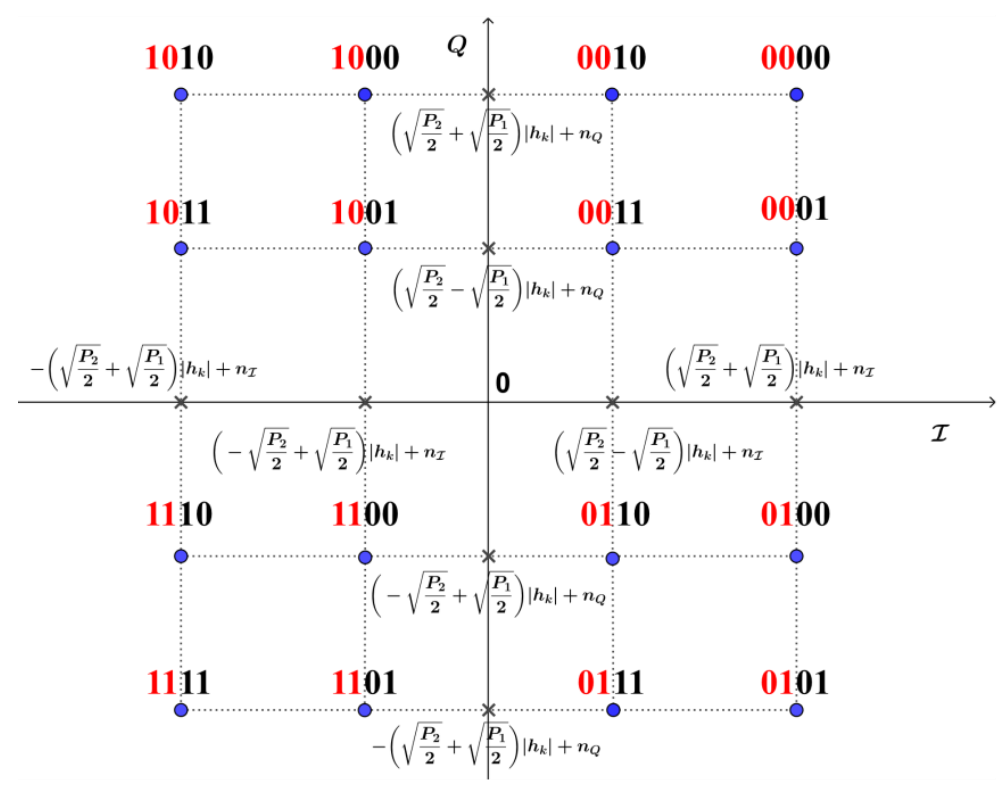

Figure 4. Reference constellation at the receiver when using MUST cat.1 


\subsection{BER of Far User}

As seen in Figure 4, the first bit of UE2, which relies only on the in-phase component, will be detected erroneously when one of these following cases occurs.

$$
\begin{aligned}
& n_{I}-\left(\sqrt{\frac{P_{2}}{2}}+\sqrt{\frac{P_{1}}{2}}\right)\left|h_{2}\right| \geq 0 \\
& n_{I}-\left(\sqrt{\frac{P_{2}}{2}}-\sqrt{\frac{P_{1}}{2}}\right)\left|h_{2}\right| \geq 0 .
\end{aligned}
$$

Considering the second bit of UE2, which depends only on the quadrature component of the signal, we see that the error cases are identical to (4.1) and (4.2). Assume the prior probability of 0 's and 1's bit is equal, so the prior probability of each symbol is also equal. The symmetry between in-phase and quadrature components of the symbol allows us to investigate only one of them. Therefore, the variance of considered AWGN now is only half of the total noise, e.g., $n_{I} \square \mathrm{N}\left(0, \frac{N_{0}}{2}\right)$. By considering maximum likelihood detection rule, scaling $Q($.$) function, the$ error probability of the FU symbols is

$$
P_{2}(e)=\frac{1}{2} Q\left(\frac{\left(\sqrt{\frac{P_{2}}{2}}+\sqrt{\frac{P_{1}}{2}}\right)\left|h_{2}\right|}{\sqrt{\frac{N_{0}}{2}}}\right)+\frac{1}{2} Q\left(\frac{\left(\sqrt{\frac{P_{2}}{2}}-\sqrt{\frac{P_{1}}{2}}\right)\left|h_{2}\right|}{\sqrt{\frac{N_{0}}{2}}}\right) .
$$

Define some notations as

$$
\begin{array}{ll}
\gamma_{A}=\frac{\left(\sqrt{P_{2}}+\sqrt{P_{1}}\right)^{2}\left|h_{2}\right|^{2}}{N_{0}} & \overline{\gamma_{A}}=\frac{\left(\sqrt{P_{2}}+\sqrt{P_{1}}\right)^{2} \mathrm{E}\left[\left|h_{2}\right|^{2}\right]}{N_{0}} \\
\gamma_{B}=\frac{\left(\sqrt{P_{2}}-\sqrt{P_{1}}\right)^{2}\left|h_{2}\right|^{2}}{N_{0}} & \overline{\gamma_{B}}=\frac{\left(\sqrt{P_{2}}-\sqrt{P_{1}}\right)^{2} \mathrm{E}\left[\left|h_{2}\right|^{2}\right]}{N_{0}} .
\end{array}
$$

In the case of quasi-static Rayleigh channel, using [10, 20, 22] with (4.4), the average BER expression of UE2 is defined as

$$
\overline{P_{2}(e)}=\frac{1}{4}\left[\left(1-\sqrt{\frac{\overline{\gamma_{A}}}{\overline{\gamma_{A}}+2}}\right)+\left(1-\sqrt{\frac{\overline{\gamma_{B}}}{\overline{\gamma_{B}}+2}}\right)\right] .
$$

\subsection{BER of Near User}

Based on [10-13], this section proposes an approach to derive BER of NU. Due to the SIC process, NU detection must be considered under the condition of FU detection. On the other hand, in Figure 4, if the second bit of FU is in error, it has no effect on the decision of NU first bit. Hence, there are two situations: The FU first bit is error-free and in error.

Case 1: When FU is error-free at NU, from Figure 4, yields: 
International Journal of Computer Networks \& Communications (IJCNC) Vol.13, No.6, November 2021

$$
\begin{aligned}
& P_{2 a}(\text { correct })=\operatorname{Pr}\left(n_{I} \leq\left(\sqrt{\frac{P_{2}}{2}}+\sqrt{\frac{P_{1}}{2}}\right)\left|h_{1}\right|\right) \\
& P_{2 b}(\text { correct })=\operatorname{Pr}\left(n_{I} \leq\left(\sqrt{\frac{P_{2}}{2}}-\sqrt{\frac{P_{1}}{2}}\right)\left|h_{1}\right|\right) .
\end{aligned}
$$

After SIC process, the reference constellation for NU is given in Figure $5[12,13]$

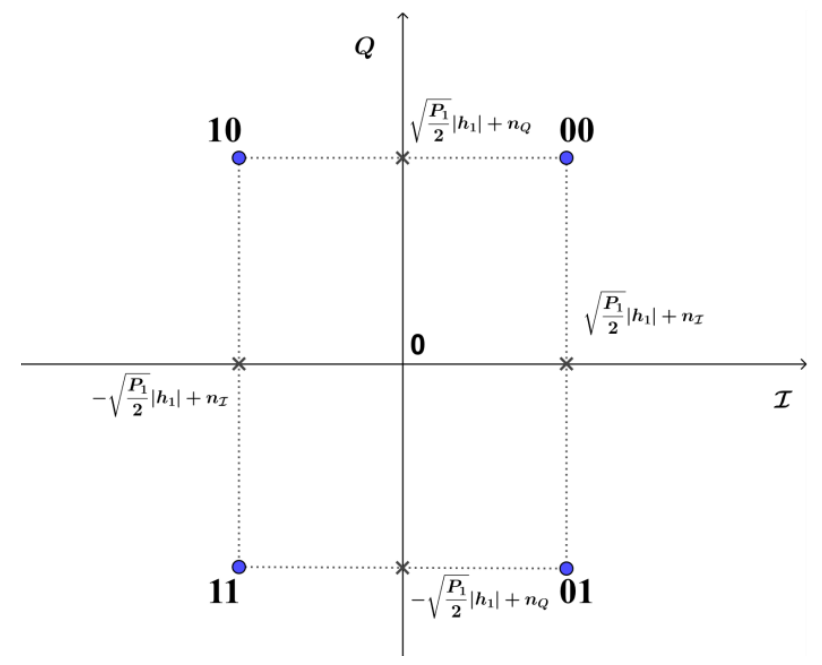

Figure 5. Reference constellation for NU after SIC when FU is error-free

From (4.6), (4.7) and 5, the error probabilities of NU are conditional probabilities as

$$
\begin{gathered}
P_{1}\left(e_{1} \mid \text { correct }_{2}\right)=\operatorname{Pr}\left(n_{I} \geq \sqrt{\frac{P_{1}}{2}}\left|h_{1}\right|\left|n_{I} \leq\left(\sqrt{\frac{P_{2}}{2}}+\sqrt{\frac{P_{1}}{2}}\right)\right| h_{1} \mid\right) \\
P_{1}\left(e_{2} \mid \text { correct }_{2}\right)=\operatorname{Pr}\left(n_{I} \leq-\sqrt{\frac{P_{1}}{2}}\left|h_{1}\right|\left|n_{I} \leq\left(\sqrt{\frac{P_{2}}{2}}-\sqrt{\frac{P_{1}}{2}}\right)\right| h_{1} \mid\right) .
\end{gathered}
$$

Applying Bayes' theorem, we have the total error probability of NU if FU is detected correctly

$$
\begin{aligned}
P_{1}\left(\text { correct }_{2}\right) & =\frac{1}{2}\left[P_{2 a}(\text { correct }) P_{1}\left(e_{1} \mid \text { correct }_{2}\right)+P_{2 b}(\text { correct }) P_{1}\left(e_{2} \mid \text { correct }_{2}\right)\right] \\
& =\frac{1}{2}\left[\operatorname{Pr}\left(\sqrt{\frac{P_{1}}{2}}\left|h_{1}\right| \leq n_{I} \leq\left(\sqrt{\frac{P_{2}}{2}}+\sqrt{\frac{P_{1}}{2}}\right)\left|h_{1}\right|\right)+\operatorname{Pr}\left(n_{I} \leq-\sqrt{\frac{P_{1}}{2}}\left|h_{1}\right|\right)\right] \\
& =Q\left(\frac{\sqrt{P_{1}}\left|h_{1}\right|}{\sqrt{N_{0}}}\right)-\frac{1}{2} Q\left(\frac{\sqrt{P_{2}}+\sqrt{P_{1}}}{\sqrt{N_{0}}}\left|h_{1}\right|\right) .
\end{aligned}
$$

Case 2: When FU is in error at NU, from Figure 4, we have:

$$
P_{2 a}(\text { error })=\operatorname{Pr}\left(n_{I} \geq\left(\sqrt{\frac{P_{2}}{2}}+\sqrt{\frac{P_{1}}{2}}\right)\left|h_{1}\right|\right)
$$


International Journal of Computer Networks \& Communications (IJCNC) Vol.13, No.6, November 2021

$$
P_{2 b}(\text { error })=\frac{1}{2} \operatorname{Pr}\left(n_{I} \geq\left(\sqrt{\frac{P_{2}}{2}}-\sqrt{\frac{P_{1}}{2}}\right)\left|h_{1}\right|\right) .
$$

Assume that the first bit of UE2 being transmitted is 0' but detected as 1', after the SIC process, the reference constellation of NU is given in Figure 6.

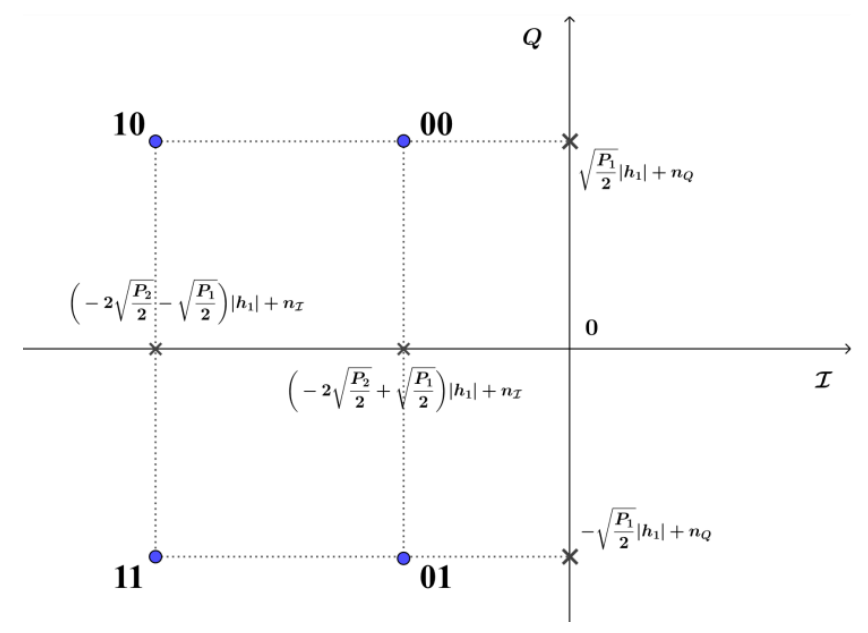

Figure 6. Reference constellation for NU after SIC when FU is erroneously detected

From (4.11), (4.12) and Figure 6, the error probability of NU when FU is in error consists of

$$
\begin{aligned}
& P_{1}\left(e_{1} \mid \text { error }_{2}\right)=\operatorname{Pr}\left(n_{I} \geq \frac{2 \sqrt{P_{2}}+\sqrt{P_{1}}}{\sqrt{2}}\left|h_{1}\right|\left|n_{I} \geq\left(\sqrt{\frac{P_{2}}{2}}+\sqrt{\frac{P_{1}}{2}}\right)\right| h_{1} \mid\right) \\
& P_{1}\left(e_{2} \mid \text { error }_{2}\right)=\operatorname{Pr}\left(n_{I} \leq \frac{2 \sqrt{P_{2}}-\sqrt{P_{1}}}{\sqrt{2}}\left|h_{1}\right|\left|n_{I} \geq\left(\sqrt{\frac{P_{2}}{2}}-\sqrt{\frac{P_{1}}{2}}\right)\right| h_{1} \mid\right) .
\end{aligned}
$$

In a similar way to (4.10), the total error probability of NU when FU is erroneously detected is obtained as

$$
\begin{aligned}
P_{1}\left(\text { error }_{2}\right) & =\frac{1}{2}\left[P_{2 a}(\text { error }) P_{1}\left(e_{1} \mid \text { error }_{2}\right)+P_{2 b}(\text { error }) P_{1}\left(e_{2} \mid \text { error }_{2}\right)\right] \\
= & \frac{1}{2}\left[\operatorname{Pr}\left(n_{I} \geq \frac{2 \sqrt{P_{2}}+\sqrt{P_{1}}}{\sqrt{2}}\left|h_{1}\right|\right)+\operatorname{Pr}\left(\frac{\sqrt{P_{2}}-\sqrt{P_{1}}}{\sqrt{2}}\left|h_{1}\right| \leq n_{I} \leq \frac{2 \sqrt{P_{2}}-\sqrt{P_{1}}}{\sqrt{2}}\left|h_{1}\right|\right)\right] \\
= & \frac{1}{2} Q\left(\frac{2 \sqrt{P_{2}}+\sqrt{P_{1}}}{\sqrt{N_{0}}}\left|h_{1}\right|\right)+\frac{1}{2} Q\left(\frac{\sqrt{P_{2}}-\sqrt{P_{1}}}{\sqrt{N_{0}}}\left|h_{1}\right|\right)-\frac{1}{2} Q\left(\frac{2 \sqrt{P_{2}}-\sqrt{P_{1}}}{\sqrt{N_{0}}}\left|h_{1}\right|\right) .
\end{aligned}
$$

From (4.10) and (4.15), the total error probability of NU is followed by 
International Journal of Computer Networks \& Communications (IJCNC) Vol.13, No.6, November 2021

$$
\begin{aligned}
P_{1}(e)= & P_{1}\left(\text { correct }_{2}\right)+P_{1}\left(\text { error }_{2}\right) \\
= & Q\left(\frac{\sqrt{P_{1}}\left|h_{1}\right|}{\sqrt{N_{0}}}\right)+\frac{1}{2}\left[Q\left(\frac{\sqrt{P_{2}}-\sqrt{P_{1}}}{\sqrt{N_{0}}}\left|h_{1}\right|\right)-Q\left(\frac{\sqrt{P_{2}}+\sqrt{P_{1}}}{\sqrt{N_{0}}}\left|h_{1}\right|\right)\right] \\
& +\frac{1}{2}\left[Q\left(\frac{2 \sqrt{P_{2}}+\sqrt{P_{1}}}{\sqrt{N_{0}}}\left|h_{1}\right|\right)-Q\left(\frac{2 \sqrt{P_{2}}-\sqrt{P_{1}}}{\sqrt{N_{0}}}\left|h_{1}\right|\right)\right] .
\end{aligned}
$$

Define some notations

$$
\begin{array}{ccc}
\gamma_{C}=\frac{P_{1}\left|h_{1}\right|^{2}}{N_{0}} & \overline{\gamma_{C}}=\frac{P_{1} \times E\left[\left|h_{1}\right|^{2}\right]}{N_{0}} \\
\gamma_{D}=\frac{\left(\sqrt{P_{2}}-\sqrt{P_{1}}\right)^{2}\left|h_{1}\right|^{2}}{N_{0}} & \overline{\gamma_{D}}=\frac{\left(\sqrt{P_{2}}-\sqrt{P_{1}}\right)^{2} E\left[\left|h_{1}\right|^{2}\right]}{N_{0}} \\
\gamma_{E}=\frac{\left(\sqrt{P_{2}}+\sqrt{P_{1}}\right)^{2}\left|h_{1}\right|^{2}}{N_{0}} & \overline{\gamma_{E}}=\frac{\left(\sqrt{P_{2}}+\sqrt{P_{1}}\right)^{2} E\left[\left|h_{1}\right|^{2}\right]}{N_{0}} \\
\gamma_{F}=\frac{\left(2 \sqrt{P_{2}}+\sqrt{P_{1}}\right)^{2}\left|h_{1}\right|^{2}}{N_{0}} & \overline{\gamma_{F}}=\frac{\left(2 \sqrt{P_{2}}+\sqrt{P_{1}}\right)^{2} E\left[\left|h_{1}\right|^{2}\right]}{N_{0}} \\
\gamma_{G}=\frac{\left(2 \sqrt{P_{2}}-\sqrt{P_{1}}\right)^{2}\left|h_{1}\right|^{2}}{N_{0}} & \overline{\gamma_{G}}=\frac{\left(2 \sqrt{P_{2}}-\sqrt{P_{1}}\right)^{2} E\left[\left|h_{1}\right|^{2}\right]}{N_{0}} . .
\end{array}
$$

Hence, the average BER of NU over quasi-static Rayleigh channel is expressed as

$$
\overline{P_{1}(e)}=\frac{1}{2}\left(1-\sqrt{\frac{\overline{\gamma_{C}}}{\overline{\gamma_{C}}+2}}\right)+\frac{1}{4}\left(-\sqrt{\frac{\overline{\gamma_{D}}}{\overline{\gamma_{D}}+2}}+\sqrt{\frac{\overline{\gamma_{E}}}{\overline{\gamma_{E}}+2}}-\sqrt{\frac{\overline{\gamma_{F}}}{\overline{\gamma_{F}}+2}}+\sqrt{\frac{\overline{\gamma_{G}}}{\overline{\gamma_{G}}+2}}\right) .
$$

\section{BER IN MUST CATEGORY 2}

Figure 7 shows the reference constellation to detect signals at each UE when using MUST category 2. Red bits denote FU's bits, and black bits are NU's bits. The order of bits is ascending, where the most significant bit is leftmost and is stated as the first bit. Gray-coded mapping is conserved for adjacent symbols. 
International Journal of Computer Networks \& Communications (IJCNC) Vol.13, No.6, November 2021

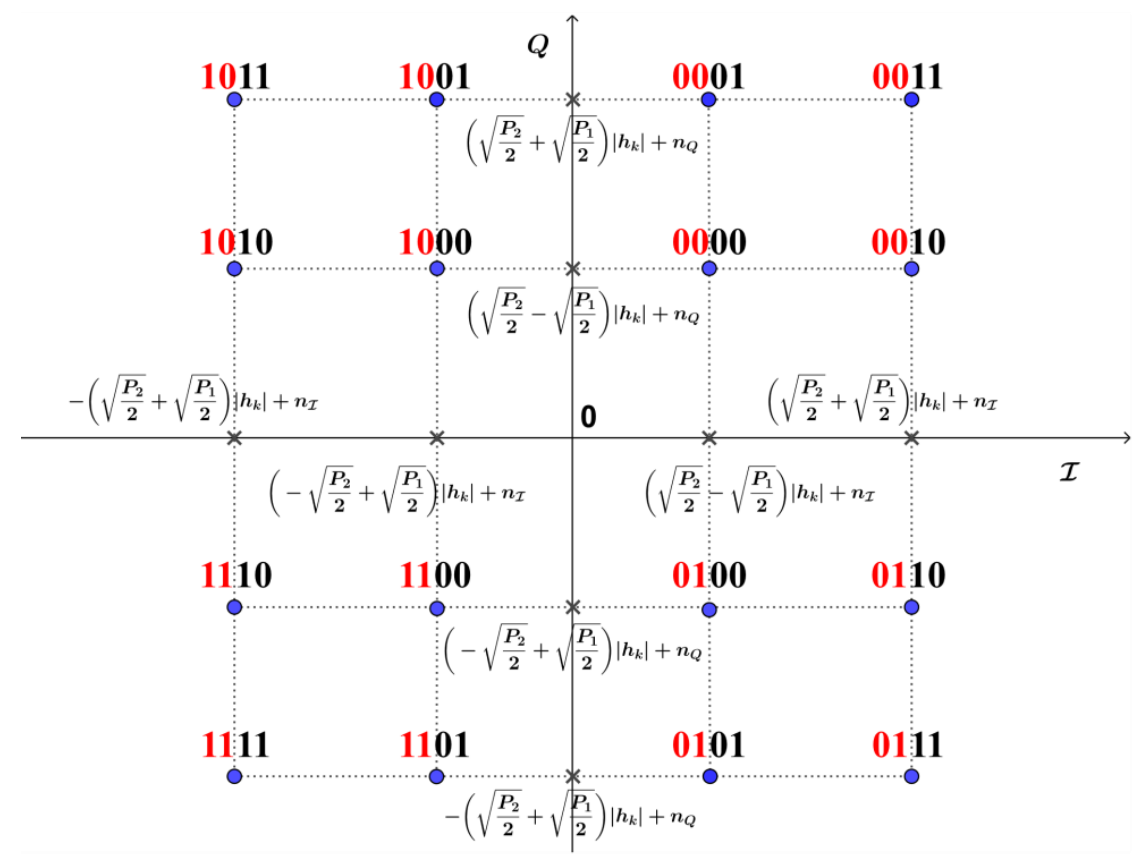

Figure 7. Reference constellation at the receiver when using MUST cat.2

\subsection{BER of Far User}

For MUST cat.2, from Figure 7, mapping rules for FU's bits remains unchanged comparing to MUST cat.1. Therefore, BER expressions of UE2 in this case are identical to (4.3) and (4.5).

\subsection{BER of Near User}

In this section, authors proposed another approach to analyze BER of NU, different from Section 4.2 and [10-13]. For UE1, error probabilities depend on the last two bits of a symbol. As a result of constellation symmetry and equal prior probabilities, the third and fourth bits are the same in error probabilities. The third bit is in error when one of these cases happens

$$
\begin{array}{ll}
-\sqrt{\frac{P_{2}}{2}}\left|h_{1}\right| \leq n_{I}+\frac{-\sqrt{P_{2}}-\sqrt{P_{1}}}{\sqrt{2}}\left|h_{1}\right| \leq 0 & n_{I}+\frac{-\sqrt{P_{2}}+\sqrt{P_{1}}}{\sqrt{2}}\left|h_{1}\right| \leq-\sqrt{\frac{P_{2}}{2}}\left|h_{1}\right| \\
0 \leq n_{I}+\frac{-\sqrt{P_{2}}-\sqrt{P_{1}}}{\sqrt{2}}\left|h_{1}\right| \leq \sqrt{\frac{P_{2}}{2}}\left|h_{1}\right| & n_{I}+\frac{-\sqrt{P_{2}}+\sqrt{P_{1}}}{\sqrt{2}}\left|h_{1}\right| \geq \sqrt{\frac{P_{2}}{2}}\left|h_{1}\right| .
\end{array}
$$

The error probability of NU calculated from (5.1) is

$$
P_{1}(e)=P_{b 3}(e)=Q\left(\frac{\sqrt{P_{1}}\left|h_{1}\right|}{\sqrt{N_{0}}}\right)+\frac{1}{2}\left[Q\left(\frac{2 \sqrt{P_{2}}-\sqrt{P_{1}}}{\sqrt{N_{0}}}\left|h_{1}\right|\right)-Q\left(\frac{2 \sqrt{P_{2}}+\sqrt{P_{1}}}{\sqrt{N_{0}}}\left|h_{1}\right|\right)\right] .
$$

And the average BER expression of NU over the quasistatic Rayleigh channel is given by (5.3), with notation in (4.17) 
International Journal of Computer Networks \& Communications (IJCNC) Vol.13, No.6, November 2021

$$
P_{1}(e)=\frac{1}{2}\left(1-\sqrt{\frac{\overline{\gamma_{C}}}{\overline{\gamma_{C}}+2}}\right)+\frac{1}{4}\left(\sqrt{\frac{\overline{\gamma_{F}}}{\overline{\gamma_{F}}}+2}-\sqrt{\frac{\overline{\gamma_{G}}}{\overline{\gamma_{G}}+2}}\right) .
$$

If we use the above approach for MUST cat.1, UE1 BER expressions obtain the same result as (4.16) and (4.18)[12, 13]. Compare (4.16) and (5.2) or (4.18) and (5.3), a BER expression of MUST cat. 2 has only three out of five components of MUST cat.1, hopefully leading to lower BER.

\section{BER IN MIMO-NOMA}

As derived in Section 3.2, we have the equalized signal, more specifically expressed as[19, 21]

$$
\tilde{\mathbf{m}}=\mathbf{m}+\tilde{\mathbf{z}}_{\mathbf{Z F}}=\left[\begin{array}{l}
m_{1} \\
m_{2}
\end{array}\right]+\left(\overline{\mathbf{h}}^{H} \overline{\mathbf{h}}\right)^{-1} \overline{\mathbf{h}}^{H} \mathbf{z}
$$

Each receiving antenna has two data streams that are perfectly separated when the channel is not correlated with the ideal ZF. Now consider the effect of ZF on AWGN. We have the singular value decomposition (SVD) for the MIMO $2 \times 2$ channel is $\overline{\mathbf{h}}=\mathbf{U} \boldsymbol{\Sigma} \mathbf{V}^{H}$, where $\mathbf{U} \in \square^{2 \times 2}$ and $\mathbf{V} \in \square^{2 \times 2}$ are unitary matrices, $\boldsymbol{\Sigma} \in \square^{2 \times 2}$ is a rectangular matrix whose diagonal elements are nonnegative real numbers and whose off-diagonal elements are zero. The diagonal elements of $\boldsymbol{\Sigma}$ are the singular values of the matrix $\overline{\mathbf{h}}$, denoting them as $\lambda_{1}, \lambda_{2}$, so $\lambda_{1}{ }^{2}$ and $\lambda_{2}{ }^{2}$ are eigen values. Then, (6.1) can be rewritten

$$
\begin{aligned}
\tilde{\mathbf{m}} & =\left[\begin{array}{l}
m_{1} \\
m_{2}
\end{array}\right]+\left[\left(\mathbf{U} \boldsymbol{\Sigma} \mathbf{V}^{H}\right)^{H} \mathbf{U} \boldsymbol{\Sigma} \mathbf{V}^{H}\right]^{-1}\left(\mathbf{U} \boldsymbol{\Sigma} \mathbf{V}^{H}\right)^{H} \mathbf{z} \\
& =\left[\begin{array}{l}
m_{1} \\
m_{2}
\end{array}\right]+\mathbf{V} \boldsymbol{\Sigma}^{-1} \mathbf{U}^{H} \mathbf{z} .
\end{aligned}
$$

From (6.2), for uncorrelated MIMO channels, eigenvectors are linearly independent leading to their singular values are non-zero and non-repeated, the expected value of noise power at fixed eigen values is given as [21]

$$
E\left[\left|\tilde{\mathbf{z}}_{\mathbf{Z F}}\right|_{\text {МIMO }}^{2}\right]=\sum_{i=1}^{2} \frac{\sigma_{z}^{2}}{\lambda_{i}^{2}}=\frac{\sigma_{z}^{2}}{\lambda_{1}^{2}}+\frac{\sigma_{z}^{2}}{\lambda_{2}^{2}}
$$

If $\lambda_{1}^{2} \square \lambda_{2}^{2}$, equation (6.3) can be approximated

$$
E\left[\left|\tilde{\mathbf{z}}_{\mathrm{ZF}}\right|_{\mathrm{MIMO}}^{2}\right]=\sum_{i=1}^{2} \frac{\sigma_{z}^{2}}{\lambda_{i}^{2}} \approx \frac{\sigma_{z}^{2}}{\lambda_{1}^{2}} .
$$

From (6.4), we can see that each antenna in the introduced MIMO encounters the same effect as SISO channel with the corresponding eigen value $\lambda_{1}^{2}$. If the condition number of the channel matrix is getting larger than a threshold, then the minimum singular value is very small, which makes the approximation be more precise. For MIMO $N_{T} \times N_{R}$ channel matrix where $N_{T}=N_{R}$ denoting the number of transmit and receive antennas respectively, $\lambda_{\min }^{2}=\min \left(\lambda_{1}^{2}, \lambda_{2}^{2}, \ldots, \lambda_{N_{T}}^{2}\right)$, followed by (6.5) 
International Journal of Computer Networks \& Communications (IJCNC) Vol.13, No.6, November 2021

$$
E\left[\left|\tilde{\mathbf{z}}_{\mathrm{ZF}}\right|_{\mathrm{MIMO}}^{2}\right]=\sum_{i=1}^{N_{T}} \frac{\sigma_{z}^{2}}{\lambda_{i}^{2}} \approx \frac{\sigma_{z}^{2}}{\lambda_{\text {min }}^{2}} .
$$

\section{NUMERICAL AND SimUlation RESUltS}

In this section, the authors used Monte Carlo simulation over independent symbols to verify all proposed expressions, under different power allocation ratios and MUST types corresponding to the model in Figure 1 and Figure 2. Simulation parameters are specified in Table 1.

Table 1. Simulation parameters for single tap fading channels

\begin{tabular}{|l|l|}
\hline Parameter & Value \\
\hline UE1's average channel gain & $g_{1}=\mathrm{E}\left[\left|\mathbf{h}_{1}\right|^{2}\right]=1$ \\
\hline UE2's average channel gain & $g_{2}=\mathrm{E}\left[\left|\mathbf{h}_{2}\right|^{2}\right]=0.5$ \\
\hline Power allocation ratio & $\alpha=\{0.2 ; 0.3\}$ \\
\hline Single antenna transmission power & $P_{S}=1$ \\
\hline Channel information & Perfectly known at receivers \\
\hline Equalization method & ZF \\
\hline Fading channel & $\begin{array}{l}\text { Single-tap Rayleigh } \\
\text { SISO, uncorrelated MIMO }\end{array}$ \\
\hline
\end{tabular}

Figure 8 shows the BER results when using MUST cat. 1 in SISO-NOMA systems where $\alpha=0.2$ and $\alpha=0.3$, corresponding to $\left(P_{1} ; P_{2}\right)=(0.2 ; 0.8)$ and $\left(P_{1} ; P_{2}\right)=(0.3 ; 0.7)$ respectively. All proposed expressions perfectly match the simulation results for both cases, as equation (4.5) for UE2 and equation (4.18) for UE1. Under given channel conditions, BER of two users in $\alpha=0.2$ is better than $\alpha=0.3$. At optimum power allocation, if increasing $\alpha$, which decreases UE2's power allocation coefficient, it leads to the degradation of the BER in UE2. In such a case, although UE1 is allocated more power, its BER is not better but on the trend of getting worse, the reason is that UE1's symbol detection depends on UE2's decoding.

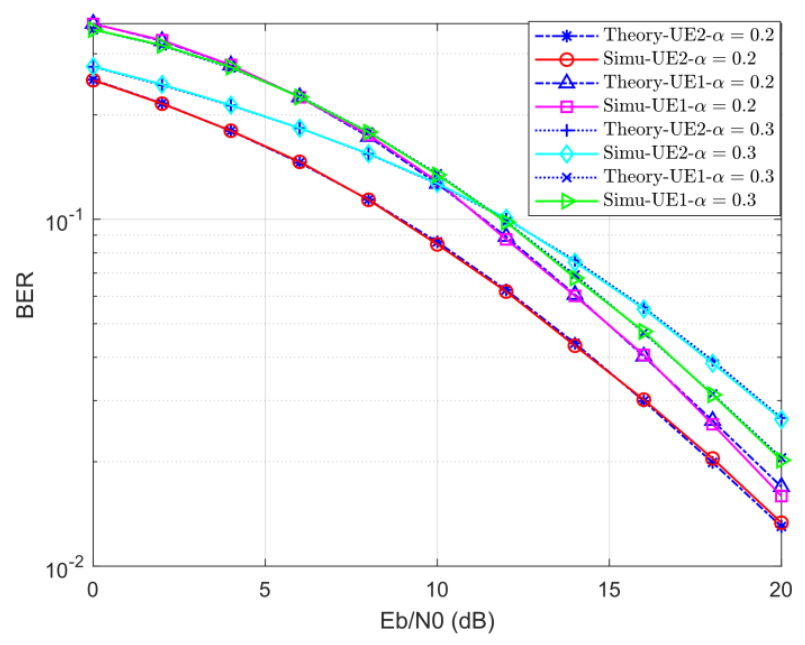

Figure 8. BER of two users in SISO-NOMA using MUST cat.1 with different power allocation ratios 
International Journal of Computer Networks \& Communications (IJCNC) Vol.13, No.6, November 2021

Figure 9 changes to MUST cat. 2 in MIMO-NOMA systems with $\alpha=0.2$ and $\alpha=0.3$. Results show that MIMO has the same BER performance as SISO under certain restraints, with BER of UE1 using equation (5.3), while UE2 using (4.5). Using MUST cat. 2 with $\alpha=0.3$, BER of UE1 is almost superior to UE2 when $E_{b} / N_{0}$ is larger than $2 \mathrm{~dB}$, while in the case of $\alpha=0.2$, to achieve that, $E_{b} / N_{0}$ needs to be larger than $15 \mathrm{~dB}$. The reason for this is MUST cat.2 ensures Gray-coded mapping for composite constellations maximum a single-bit error for sufficient $E_{b} / N_{0}$, if allocating more power to UE1, we are "protecting" UE1 more than UE2. Compared to Figure 8, MUST cat. 2 is also better than MUST cat.1.

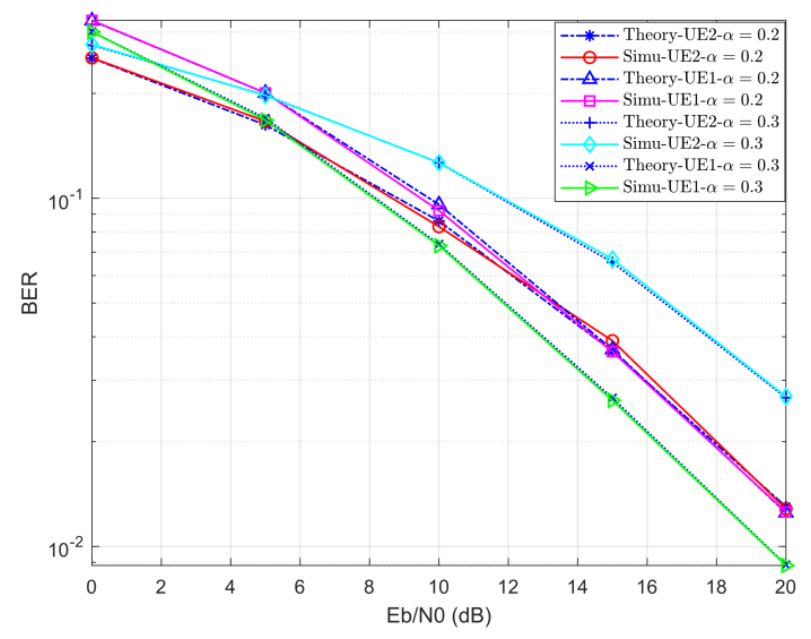

Figure 9. BER of two users in MIMO-NOMA using MUST cat.2 with different power allocation ratios

Figure 10 shows the results when the SISO channel has multiple taps, we need to scale the analytical expression SNR, but due to error in limited ZF equalization causing ISI, simulations barely fit derived BER formulas. When the system is combined with OFDM (see Table 2 for parameters), BER curves match the expressions due to ISI elimination in cyclic prefixes. It is noteworthy that SNR must be scaled for OFDM signals.

Table 2. Simulation parameters for two-tap fading channels

\begin{tabular}{|l|l|}
\hline Parameter & Value \\
\hline UE1's average channel gain & $g_{1}=\mathrm{E}\left[\left|\mathbf{h}_{1}\right|^{2}\right]=1$ \\
\hline UE2's average channel gain & $g_{2}=\mathrm{E}\left[\left|\mathbf{h}_{2}\right|^{2}\right]=0.5$ \\
\hline Power allocation ratio & $\alpha=0.2$ \\
\hline Single antenna transmission power & $P_{S}=1$ \\
\hline Number of FFT points & 64 \\
\hline Number of data subcarriers & 52 \\
\hline Number of zeros & 1 DC, 11 guard bands \\
\hline Number of cyclic prefixes & 16 \\
\hline Channel information & Perfectly known at receivers \\
\hline Equalization method & ZF \\
\hline Fading channel & Rayleigh SISO 2 taps \\
\hline
\end{tabular}




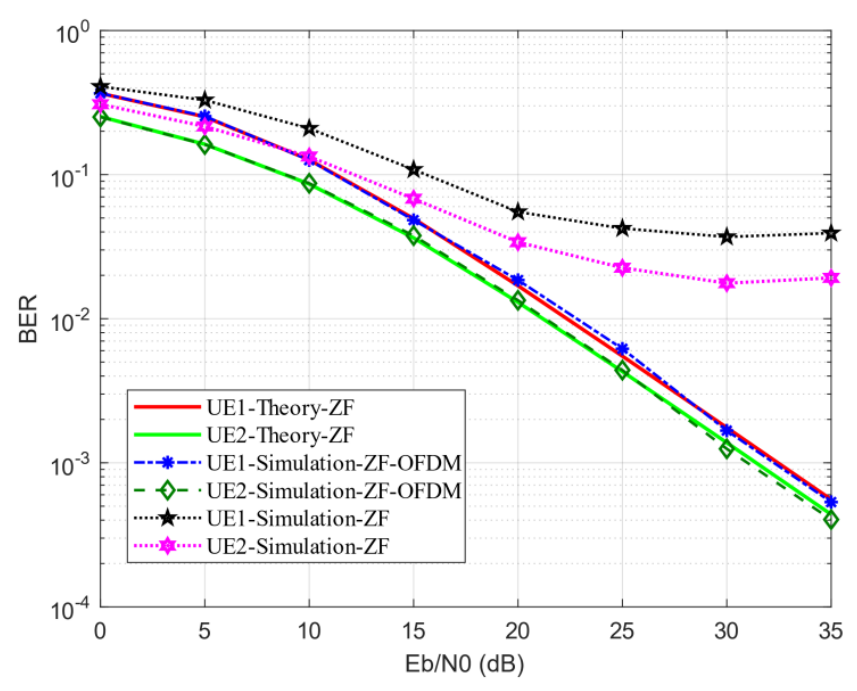

Figure 10. BER of two NOMA users in SISO-ZF-OFDM and SISO-ZF systems using MUST cat.1

\section{CONClusion ANd Future Work}

This research has introduced BER expressions for downlink MIMO-NOMA-ZF systems using a SIC receiver, serving two users with QPSK modulation over an uncorrelated quasi-static Rayleigh channel. Simulation results are perfectly matched with analytical expressions, not only in different power allocation ratios but also MUST type 1 and 2 for signal generations. The results show that MUST type 2 has better performance than type 1 due to Gray-mapping rules, and when combined with OFDM, the system can minimize ISI, the simulations fit well with the proposed theory. An approach to compare the performance between SISO and MIMO in the NOMA system has also been clarified.

When the channel is bad or there is no need for high data rate, we can consider using diversity instead, such as space-time coding, or develop a machine-learning detection system to ensure integrity. Future work can be conducted for higher modulation orders, antenna beam forming, optimal power allocation, MMSE or different equalization techniques, increasing the number of users, having non-linear effects, and over correlated channel models.

\section{CONFLICT OF INTEREST}

The authors declare no conflict of interest.

\section{ACKNOWLEDGMENT}

This research is funded by Vietnam National University HoChiMinh City (VNU-HCM) under grant numberC2019-18-32.

\section{REFERENCES}

[1] Y. Liu, Z. Qin, M. Elkashlan, Z. Ding, A. Nallanathan, and L. Hanzo, "Nonorthogonal Multiple Access for 5G and Beyond," Proceedings of the IEEE, vol. 105, no. 12, pp. 2347-2381, 2017, doi: 10.1109/JPROC.2017.2768666.

[2] Y. Liu, W. Yi, Z. Ding, X. Liu, O. Dobre, and N. Al-Dhahir, Application of NOMA in 6G Networks: Future Vision and Research Opportunities for Next Generation Multiple Access. 2021. 
[3] K. Dang, H. Ngo, and N. Nguyen, "Performance of non-orthogonal multiple access downlink system using the Log-Likelihood ratio," Science and Technology Development Journal - Natural Sciences, vol. 4, no. 3, pp. 621-632, 2020, doi: 10.32508/stdjns.v4i3.662.

[4] Narengerile and J. Thompson, "Deep Learning for Signal Detection in Non-Orthogonal Multiple Access Wireless Systems," in 2019 UK/ China Emerging Technologies (UCET), 21-22 Aug. 2019 2019, pp. 1-4, doi: 10.1109/UCET.2019.8881888.

[5] J. M. Kang, I. M. Kim, and C. J. Chun, "Deep Learning-Based MIMO-NOMA With Imperfect SIC Decoding," IEEE Systems Journal, vol. 14, no. 3, pp. 3414-3417, 2020, doi: 10.1109/JSYST.2019.2937463.

[6] A. Agarwal, R. Chaurasiya, S. Rai, and A. K. Jagannatham, "Outage Probability Analysis for NOMA Downlink and Uplink Communication Systems With Generalized Fading Channels," IEEE Access, vol. 8, pp. 220461-220481, 2020, doi: 10.1109/ACCESS.2020.3042993.

[7] J. A. Oviedo and H. R. Sadjadpour, "A Fair Power Allocation Approach to NOMA in Multiuser SISO Systems," IEEE Transactions on Vehicular Technology, vol. 66, no. 9, pp. 7974-7985, 2017, doi: 10.1109/TVT.2017.2689000.

[8] Q. Sun, S. Han, I. C, and Z. Pan, "On the Ergodic Capacity of MIMO NOMA Systems," IEEE Wireless Communications Letters, vol. 4, no. 4, pp. 405-408, 2015, doi: 10.1109/LWC.2015.2426709.

[9] X. Wang, F. Labeau, and L. Mei, "Closed-Form BER Expressions of QPSK Constellation for Uplink Non-Orthogonal Multiple Access," IEEE Communications Letters, vol. 21, no. 10, pp. 2242-2245, 2017, doi: 10.1109/LCOMM.2017.2720583.

[10] F. Kara and H. Kaya, "BER performances of downlink and uplink NOMA in the presence of SIC errors over fading channels," IET Communications, vol. 12, no. 15, pp. 1834-1844, 2018, doi: 10.1049/iet-com.2018.5278.

[11] T. Assaf, A. Al-Dweik, M. E. Moursi, and H. Zeineldin, "Exact BER Performance Analysis for Downlink NOMA Systems Over Nakagami-m Fading Channels," IEEE Access, vol. 7, pp. 134539134555, 2019, doi: 10.1109/ACCESS.2019.2942113.

[12] J. Garnier, A. Fabre, H. Farès, and R. Bonnefoi, "On the Performance of QPSK Modulation Over Downlink NOMA: From Error Probability Derivation to SDR-Based Validation," IEEE Access, vol. 8, pp. 66495-66507, 2020, doi: 10.1109/ACCESS.2020.2983299.

[13] T. Assaf, A. J. Al-Dweik, M. S. E. Moursi, H. Zeineldin, and M. Al-Jarrah, "Exact Bit Error-Rate Analysis of Two-User NOMA Using QAM With Arbitrary Modulation Orders," IEEE Communications Letters, vol. 24, no. 12, pp. 2705-2709, 2020, doi: 10.1109/LCOMM.2020.3020161.

[14] Y. Saito, Y. Kishiyama, A. Benjebbour, T. Nakamura, A. Li, and K. Higuchi, "Non-Orthogonal Multiple Access (NOMA) for Cellular Future Radio Access," in 2013 IEEE 77th Vehicular Technology Conference (VTC Spring), 2-5 June 2013 2013, pp. 1-5, doi: 10.1109/VTCSpring.2013.6692652.

[15] E. Stergiou, C. Angelis, and S. Margariti, "Evaluation Methodology of MIMO Networks Performance over Rayleigh Fading," International journal of Computer Networks \& Communications, vol. 12, pp. 37-52, 01/31 2020, doi: 10.5121/ijcnc.2020.12103.

[16] M. Aljumaily and H. Li, "Mobility Speed Effect and Neural Network Optimization for Deep MIMO Beamforming in mmWave Networks," International Journal of Computer Networks and Communications, vol. 12, pp. 1-14, 11/24 2020, doi: 10.5121/ijcnc.2020.12601.

[17] 3GPP. "Study on Downlink Multiuser Superposition Transmission (MUST) for LTE." https://itectec.com/archive/3gpp-specification-tr-36-859/ (accessed 2021).

[18] G. Americas, Wireless Technology Evolution Towards 5G: 3GPP Release 13 to Release 15 and beyond. 2017.

[19] A. Goldsmith, Wireless Communications. Cambridge University Press, 2005.

[20] M. K. Simon and M.-S. Alouini, Digital Communication over Fading Channels, Second ed. John Wiley \& Sons, Inc., 2004.

[21] Y. S. Cho, J. Kim, W. Y. Yang, and C. G. Kang, MIMO-OFDM Wireless Communications with MATLAB. John Wiley \& Sons (Asia) Pte Ltd, 2010.

[22] I. S. Gradshteyn and I. M. Ryzhik, D. Zwillinger and V. Moll, Eds. Table of Integrals, Series, and Products, Eighth ed. 2014. 


\section{AUTHORS}

Vu Tran Hoang Son received a B.S. degree in Electronics and Telecommunications, minor in Telecommunications and Networks, from the University of Science, Vietnam National University, HoChiMinh City (VNU-HCM). He is focusing on new-generation mobile networks and wireless communications.

Dang Le Khoagraduated B.E. and Ph.D. in Radio Physics and Electronics from University of Science, Vietnam National University, HoChiMinhCity (VNU-HCM). He is the head of the Telecommunications and Networks Department, University of Science, VNU-HCM. His current research interests are in the areas of wireless communications and digital signal processing for telecommunication.
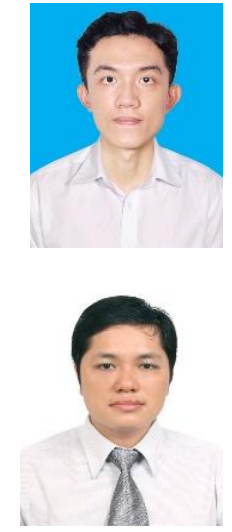